\title{
Molecular Classification of Tumors
}

National Cancer Institute

\section{Source}

National Cancer Institute. Molecular Classification of Tumors. NCI Thesaurus. Code C16090.

Challenge to research community to revolutionize the classification of human tumors, away from classification based on morphology, toward a more clinically predictive and useful classification system based on molecular characterization of tumors. (Bypass Budget) 